\title{
Protocol Amendment Signature Page
}

National Cancer Institute

\section{Source}

National Cancer Institute. Protocol Amendment Signature Page. NCI Thesaurus. Code C115718.

Records that contain both the amendments to a clinical trial's protocol and the signatures of investigators and sponsors agreeing to abide by the amended protocol. 